\title{
Epigenetic dysregulation in hematological malignancies
}

\author{
Atsushi Iwama1
}

Received: 19 October 2016 / Revised: 24 October 2016 / Accepted: 1 November 2016 / Published online: 7 November 2016

(C) The Japanese Society of Hematology 2016

Next-generation sequencing technologies have revealed mutations in a variety of epigenetic regulator genes in cancer [1], and the involvement of epigenetic dysregulation in the development of cancer is now well understood. Epigenetic dysregulation is caused not only by genetic mutations, but also by various oncogenic signals that lead to the reprogramming of transcriptional profiles in transformed cells.

The role of epigenetic gene regulation has been extensively analyzed in hematopoiesis, particularly in hematopoietic stem cells (HSCs) [2]. Analyses of mice deficient for epigenetic regulator genes have shown that epigenetic dysregulation is indeed closely associated with hematological malignancies [3]. Based on the growing body of evidence on the role of epigenetic dysregulation in the pathophysiology of cancer, a series of pharmacological agents targeting epigenetic regulators have been identified and studied for clinical use [4]. The Progress in Hematology reviews in this issue highlight the consequences of epigenetic dysregulation in hematological malignancies.

Histone methylation is a major epigenetic determinant in gene expression and is frequently dysregulated in hematological malignancies. Methylation of histone H3 lysine 4 (H3K4), which is regulated by the SET/MLL family methyltransferases, has been extensively analyzed in normal hematopoiesis and hematological malignancies. In the SET/MLL family, MLL1 is involved in chromosomal translocations in leukemia, and MLL3 and MLLA/KMT2D are also frequently implicated in hematological malignancies

Atsushi Iwama

aiwama@faculty.chiba-u.jp

1 Department of Cellular and Molecular Medicine, Graduate School of Medicine, Chiba University, 1-8-1 Inohana, Chuo-ku, Chiba 260-8670, Japan due to chromosomal deletions and somatic gene mutations, respectively. However, the consequences of gene aberrations in MLL family members are unique. Drs. Weiwei Yang and Patricia Ernst provide an overview of the role of SET/MLL family proteins in normal hematopoiesis and hematological malignancies, with an emphasis on their unique biological functions in vivo.

Of the various repressive histone marks, methylation of $\mathrm{H} 3 \mathrm{~K} 27$, which is regulated by Polycomb Repressive Complex (PRC) 2, has also been extensively analyzed. EZH2, the gene encoding an enzymatic component of PRC2, is often mutated in hematological malignancies. EZH2 has various cell type-dependent biological functions, and may function as either an oncogene or a tumor suppressor depending on the cancer type, which complicates our understanding of the consequences of EZH2 mutations. Dr. Goro Sashida and I update the latest findings on EZH2 dysregulation in hematological malignancies, and describe how EZH2 mutations affect epigenetic status and disrupt hematopoietic homeostasis.

Alteration in DNA methylation status is a characteristic epigenetic change in cancer, and one that, in hematological malignancies, is often enhanced by somatic gene mutations in either DNMT3A, a DNA methyltransferase gene, or in TET family DNA demethylating enzyme genes, most frequently TET2. Loss of TET2 results in reduced 5-hydroxymethyl cytosine $(5-\mathrm{hmC})$ levels and a resultant increase in 5-methyl cytosine (5-mC) levels. Dynamic changes in DNA methylation status have a strong impact on gene expression. Dr. Shigeru Chiba focuses on the pathophysiological role of dysregulated TET2 in hematological malignancies and underscores its dysregulation as an early event in the ontogeny of hematological malignancies.

New technologies are also uncovering abnormal nuclear topology and chromatin structure in cancer [2]. Recurrent 
mutations in cohesin complex proteins have been identified in pre-leukemic HSCs during the development of myeloid malignancies. Although cohesins are involved in chromosome separation and DNA damage repair, the roles played by dysregulated cohesin in leukemic development remain unclear. Drs. Claire Mazumdar and Ravindra Majeti provide the latest in the mechanism through which dysregulated cohesion compromises the differentiation of HSCs and induces global changes in chromatin accessibility and transcription factor activity.

The PIH reviews assembled in this issue reflect the authors' collective expertise in the epigenetics of hematopoiesis and hematological malignancies and should provide readers with a clear understanding of the state of the science in these important and exciting areas of research.
I hope that readers will find these articles useful and informative.

\section{References}

1. Feinberg AP, Koldobskiy MA, Göndör A. Epigenetic modulators, modifiers and mediators in cancer aetiology and progression. Nat Rev Genet. 2016;17:284-99.

2. Avgustinova A, Benitah SA. Epigenetic control of adult stem cell function. Nat Rev Mol Cell Biol. 2016;17:643-58.

3. Ntziachristos P, Abdel-Wahab O, Aifantis I. Emerging concepts of epigenetic dysregulation in hematological malignancies. Nat Immunol. 2016;17:1016-24.

4. Jones PA, Issa JP, Baylin S. Targeting the cancer epigenome for therapy. Nat Rev Genet. 2016;17:630-41. 